\title{
Nonparallel spatial stability of shallow water flow down an inclined plane of arbitrary slope
}

\author{
P. Bohorquez \& R. Fernandez-Feria \\ E.T.S.Ingenieros Industriales, Universidad de Málaga (Spain)
}

\begin{abstract}
Roll waves are known to occur in the frictional flow of a thin layer of water down an inclined solid surface. For a layer of constant depth, the formation of these waves on a solid plane with small slope angle have been explained as a hydrodynamic instability occurring above a critical Froude number by analyzing the temporal stability of the constant velocity flow. Here we analyze the linear, spatial stability of the shallowwater flow down an inclined plane of arbitrary slope including the first order effects of the gradients of both the velocity and the water depth. For constant water depth (parallel case) we reproduce previous results of the temporal stability analysis. Then we apply the nonparallel stability formulation to the kinematic wave approximation of the shallow-water flow down an inclined plane of arbitrary slope and characterize, analytically, the frequency and the wavelength of the most unstable waves as a function of the Froude number, slope angle, velocity and velocity gradient. We find important qualitative differences with respect to the parallel (constant depth) case. These stability results are used to discuss the numerical solution to the nonlinear shallow-water flow equations for the dam-break problem on an inclined surface of arbitrary slope. A good agreement between the waves resulting from the numerical simulations and the predictions of the stability analysis is found.
\end{abstract}

\section{INTRODUCTION}

A thin layer of water flowing down an inclined surface may undergo, in some circumstances, a transition to an oscillatory movement where a train of surface waves propagates downstream. The amplitude of these waves increases, and eventually break into the form of a train of hydraulic bores at quite regular intervals, as they propagate downstream. These so-called roll waves where first scientifically observed in open channel flows by Cornish in 1904 (Cornish 1934), and have been subsequently documented in many manmade and natural shallow water flows (Kenyon 1998; Chanson 2004). The presence of these waves is generally undesirable from the hydraulic engineer point of view because they can produce overflow from the channel sides and because excessive intermittency at the outlet. Therefore the interest in determining with precision the conditions under which they appear.

Since the early work of Jeffreys (1925), these roll waves are known to be originated by inertial instabilities of the turbulent shallow water flow in a channel. Particularly, this author analysed the linear, temporal stability of the St. Venant one-dimensional model for the uniform flow over a plane, including a drag term proportional to the square of the velocity, and found the instability condition $F r>2$, where $F r$ is the Froude number (see below for its definition). Subsequently,
Dressler (1949) confirmed this stability result and constructed finite-amplitude roll waves by piecing together smooth solutions separated by discontinuous shocks. Experiments by Brock (1967) showed the formation of roll waves for $\mathrm{Fr}>2$. Further linear stability results for the uniform turbulent flow down open inclined channel include both temporal (Brock 1967; Whitham 1974; Berlamont and Vanderstappen 1981) and spatial stability analysis (Di Cristo and Vacca 2005). All these stability results do not modify Jeffrey's basic finding that the uniform flow becomes unstable for $F r>2$.

In this work we consider the spatial stability analysis of the shallow water flow with friction down an inclined plane. The two features introduced here that have not been considered before are that the basic flow is valid for arbitrary slope of the channel, and that nonparallel effects accounting for the slow streamwise variations of both the basic flow and the perturbation are taken into account. Earlier stability analysis of the shallow water flow down an open inclined channel used the St. Venant one-dimensional shallowwater equations, valid for small slopes of the channel (Stoker 1957; Whitham 1974). Recently, Bouchut et al. (2003) and Keller (2003) have generalized these shallow water equations for arbitrary slopes of the bottom of the channel. But, for a constant (but arbitrary) slope, the equations are formally the same as the St. Venant 
equations, provided that appropriate variables are used (Savage and Hutter 1991). Therefore, this generalisation to an arbitrary slope of the inclined plane does not modify, as we shall see, the basic Jeffreys's stability result. However, we find that the inclusion of nonparallel effects in the stability analysis qualitatively modify the stability properties of the flow. Therefore, the critical parameters at which roll waves are predicted in a non-uniform flow may be substantially different from those in an uniform flow.

The structure of the paper is as follows. In the next section we formulate the spatial stability problem. We consider non-parallel effects for a general non-uniform flow down an inclined plane of arbitrary slope. Then we reproduce the known stability results for a uniform flow when non-parallel effects are neglected, but for arbitrary slope of the channel. Detailed stability properties of non-uniform flows, when non-parallel effects are included, are given for the case when the kinematic wave approximation (Whitham 1974) is used for the basic flow. These stability results are then compared with the outcome of some numerical simulations for the dam-break problem on an inclined surface of arbitrary slope, and with some available experimental results. Finally, some conclusions are drawn in the last section.

\section{FORMULATION OF THE PROBLEM}

We consider here the one-dimensional flow over a constant slope bed. In the shallow-water approximation, the dimensionless equations for the mass conservation and momentum in the direction of the flow can be written as [see Fig. 1]

$\frac{\partial \eta}{\partial t}+\frac{\partial \eta U}{\partial X}=0$

$\frac{\partial U}{\partial t}+U \frac{\partial U}{\partial X}+\cos \theta \frac{\partial \eta}{\partial X}=\sin \theta-\frac{s_{f}}{\eta}$,

where $\theta$ is the angle between the bed and the horizontal, $t$ is the time, $X$ is the coordinate along the bed, $\eta$ is the depth of the water measured along the coordinate $Y$ perpendicular to the bed, $U$ is the depth-averaged velocity component along $X$, and $s_{f}$ is a dimensionless bed friction (see below). All the magnitudes in these equations have been non-dimensionalized with respect to a length scale $\eta_{0}$, corresponding to some initial depth, and a velocity scale $U_{0} \equiv \sqrt{g \eta_{0}}$, where $g$ is the acceleration due to gravity. Equations (1)-(2) are formally the same as the classical St. Venant equations for the shallow-water in a channel with small angle of inclination $\theta$ (Stoker 1957). However, it can be shown (Savage and Hutter 1991; Bouchut et al. 2003; Keller 2003) that these equations, written in the present coordinates $X$ and $Y$ (see Fig. 1), are valid for any slope

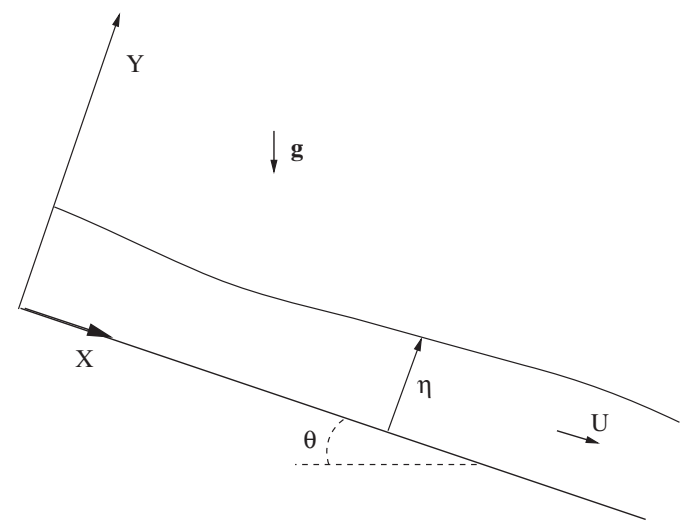

Figure 1. Sketch of coordinates and variables.

$\tan \theta$ of the constant-slope bed, not just for small channel slope, provided that the characteristic length scale of the flow in the direction of the coordinate $X$ is much greater than the characteristic length scale in the $Y$ direction.

To compute the friction term, $s_{f} \equiv\left(\tau_{b} / \rho U_{0}^{2}\right)$, where $\tau_{b}$ is the bed shear stress and $\rho$ the fluid density, we shall use the Darcy-Weisbach friction factor $f$, so that $s_{f}$ may be written as

$s_{f}=\frac{f}{8}|U| U$.

The factor $f$ is a function of the local Reynolds number, based on the velocity $U$ and the hydraulic diameter of the channel, and the relative height roughness of the bed. However, to simplify the present analysis we shall assume that the friction factor $f$ is constant in what follows.

Here we shall analyse the spatial stability of a flow governed by Eqs. (1)-(2). To that end, if $\eta=H(X, t)$ and $U=V(X, t)$ is a solution to these equation, the perturbed flow is decomposed, as usual, as the sum of the basic flow solution plus a small perturbation, $\eta^{\prime}(X, t)$ and $U^{\prime}(X, t)$,

$\eta=H+\eta^{\prime}, \quad U=V+U^{\prime}$,

where the (non-dimensional) perturbations satisfy

$\left|\eta^{\prime}\right| \ll H, \quad$ and $\quad\left|U^{\prime}\right| \ll V$.

We shall also assume that the streamwise variation of the basic flow is small,

$\left|\frac{\partial H}{\partial X}\right| \ll 1, \quad\left|\frac{\partial V}{\partial X}\right| \ll 1$.

Substituting (4) into (1)-(2), taking into account that $[H, V]$ is a solution to the equations, and neglecting 
second order terms in both the small perturbations and the streamwise derivatives of the basic flow, one is left with the following set of linear equations for the perturbations:

$\frac{\partial \eta^{\prime}}{\partial t}+\frac{\partial H U^{\prime}}{\partial X}+\frac{\partial V \eta^{\prime}}{\partial X}=0$

$\frac{\partial U^{\prime}}{\partial t}+\frac{\partial V U^{\prime}}{\partial X}+\cos \theta \frac{\partial \eta^{\prime}}{\partial X}+\frac{f V^{2}}{8 H}\left(\frac{2 U^{\prime}}{V}-\frac{\eta^{\prime}}{H}\right)=0$.

The perturbation $\mathbf{s} \equiv\left[\eta^{\prime}(X, t), U^{\prime}(X, t)\right]^{T}$, where the superscript $T$ denotes transposed vector, is decomposed in the standard form

$\mathbf{s}(X, t)=\mathbf{S}(X) \chi(X, t)$

where the complex amplitude

$\mathbf{S}(X) \equiv\left(\begin{array}{c}F(X) \\ G(X)\end{array}\right)$

depends only on the streamwise coordinate. In accordance with (6), we shall assume that $|d \mathbf{S} / d X| \ll 1$. The other part of the perturbation is of exponential form and describes the wave-like nature of the disturbance,

$\chi(X, t)=\exp \left[\int_{X_{i}}^{X} a\left(X^{\prime}\right) d X^{\prime}-i \omega t\right]$

where $X_{i}$ is an initial or reference value of the coordinate $X$. The non-dimensional, complex wavenumber $a$ is defined as

$a(X) \equiv \gamma(X)+i \alpha(X)$.

The real part $\gamma(X)$ is the local exponential growth rate, and the imaginary part $\alpha(X)$ is the local wavenumber. A non-dimensional frequency $\omega$ has also been defined in (11).

Substituting (9)-(12) into (7)-(8), the resulting set of two linear equations may be written in the matrix form

$$
\mathbf{A} \cdot \mathbf{S}+a \mathbf{B} \cdot \mathbf{S}+\mathbf{B} \cdot \frac{d \mathbf{S}}{d X}=0
$$

where

$$
\begin{gathered}
\mathbf{A}=\left(\begin{array}{cc}
-i \omega+V_{X} & H_{X} \\
-\frac{f V^{2}}{8 H^{2}} & -i \omega+V_{X}+\frac{f V}{4 H}
\end{array}\right), \\
\mathbf{B}=\left(\begin{array}{cc}
V & H \\
\cos \theta & V
\end{array}\right),
\end{gathered}
$$

and $H_{X} \equiv \frac{\partial H}{\partial X}, V_{X} \equiv \frac{\partial V}{\partial X}$.
The retained terms in (13) account for two different non-parallel effects on the stability of the perturbations: the effect of the non-parallelism of the basic flow and of the amplitude of the perturbations, and the effect of the history, or convective evolution, of the perturbations. This last effect is described by the $d / d X$ terms of the stability equation. All these effects are negligible in the parallel limit of uniform basic flow with uniform perturbations.

As it stands there is some ambiguity in the partition of the perturbations (9) into two functions of the coordinate $X$. To close the problem one has to enforce an additional normalization condition which puts some restriction on the streamwise variation of the perturbation amplitude (Bertolotti et al. 1992). We shall perform here a local spatial stability analysis (Fernandez-Feria 2000): Given a real frequency $\omega$, Eq. (13) and its $X$-derivative will be solved locally for each location $X=X_{0}$ with the normalization condition $[d a / d X]_{X=X_{0}}=0$. This condition will restrict, as required, the downstream variation of the perturbation amplitude (eigenfunction), yielding, for each $X=X_{0}$, the local growth rate and the wavenumber (or the phase speed of the disturbance). To that end, the eigenfunction $\mathbf{S}$ is expanded in a Taylor series about $X=X_{0}$, where only two terms are retained to be consistent with the approximation made in Eq. (13):

$$
\begin{gathered}
\mathbf{S}(X) \simeq \mathbf{S}\left(X_{0}\right)+\left.\left(X-X_{0}\right) \frac{d \mathbf{S}}{d X}\right|_{X=X_{0}} \\
\equiv \mathbf{S}_{0}+\left(X-X_{0}\right) \mathbf{S}_{1} .
\end{gathered}
$$

This expansion is now substituted into (13) and its $X$-derivative to obtain two equations for $\mathbf{S}_{0}$ and $\mathbf{S}_{1}$ $\left(\left|\mathbf{S}_{1}\right| \ll\left|\mathbf{S}_{0}\right|\right)$. Using the local normalization condition $[d a(X) / d X]_{X=X_{0}}=0$, one has a set of four homogeneous linear equation that may be written as

$\mathbf{F} \cdot \boldsymbol{T}=\mathbf{0}$,

where

$\mathbf{F}=\left(\begin{array}{cc}\mathbf{A}+a \mathbf{B} & \mathbf{B} \\ \mathbf{C}+a \mathbf{D} & \mathbf{E}+a \mathbf{B}\end{array}\right), \quad \boldsymbol{T} \equiv\left(\begin{array}{l}\mathbf{S}_{0} \\ \mathbf{S}_{1}\end{array}\right)$,

$\mathbf{C}=\left(\begin{array}{cc}0 & 0 \\ -\frac{f V}{4 H^{3}}\left(H V_{X}-V H_{X}\right) & \frac{f}{4 H^{2}}\left(H V_{X}-V H_{X}\right)\end{array}\right)$,

$$
\begin{aligned}
& \mathbf{D}=\left(\begin{array}{cc}
V_{X} & H_{X} \\
0 & V_{X}
\end{array}\right), \\
& \mathbf{E}=\left(\begin{array}{cc}
-i \omega+2 V_{X} & 2 H_{X} \\
-\frac{f V^{2}}{8 H^{2}} & -i \omega+2 V_{X}+\frac{f V}{4 H}
\end{array}\right) .
\end{aligned}
$$

For a given basic flow, and given $\omega$ and $X=X_{0}$ (for simplicity, in the above expressions, and in what 
follows, we write $X$ for the local value $X_{0}$ ), the homogeneous equation (17) constitutes an eigenvalue problem for the complex eigenvalue $a$ and the complex eigenfunction $\boldsymbol{T}$. That is to say, the homogeneous equation (17) has nontrivial solutions only when the determinant of $\mathbf{F}$ vanishes, yielding a dispersion relation of the form

$D\left(a, \omega ; \theta, f, V, H, V_{X}, H_{X}\right)=0$,

that determines the eigenvalue $a$.

One is interested in looking for convective instabilities propagating towards $X>0$, i.e. in the same direction as the basic flow. Thus, for a given positive value of the real frequency $\omega$, one is interested in modes whose eigenvalue $a$ has both its real and imaginary parts positive. According to (11)-(12), this ensures that the perturbation grows exponentially as it propagates towards increasing $X(\gamma>0)$, with phase velocity directed downstream (i.e. $c_{p h} \equiv \omega / \alpha>0$ ). As a matter of fact, one has to consider the sign of the group velocity,

$c_{g} \equiv \frac{\partial \omega}{\partial \alpha}$

instead of the sign of the phase velocity $c_{p h}$, in order to determine the propagation direction of the perturbations. But, as we shall see, the sign of $c_{g}$ always coincide with the sign of $c_{p h}$ in all the cases considered below (all the instabilities found are convective instabilities, with both $c_{g}$ and $c_{p h}$ positive). On the other hand, the frequency range of study can be reduced to just non-negative values because the real and imaginary parts of the linear operator $\mathbf{F}$,

$\mathbf{F}(\omega, \alpha, \gamma) \equiv \mathbf{F}_{\Re}(\gamma)+i \mathbf{F}_{\Im}(\omega, \alpha)$,

have the property

$\mathbf{F}(a, \omega)=\overline{\mathbf{F}}(\bar{a},-\omega)$,

where $\overline{(\cdot)}$ denotes the complex conjugate, and both $\mathbf{F}_{\Re}$ and $\mathbf{F}_{\Im}$ are real functions. Thus, if $a$ is the eigenvalue corresponding to the real frequency $\omega, \bar{a}$ is also an eigenvalue of the problem, corresponding to the frequency $-\omega$. So that one has to consider only nonnegative values of $\omega$ to fully analyse the linear stability of the flow.

\section{RESULTS}

\subsection{Parallel flow: Roll waves}

Before undertaking the non-parallel stability analysis of a non-uniform flow down an open inclined channel, it is convenient to consider first the simplest case of an uniform flow, thus reproducing previous stability results. This analysis will also serve as a reference for better understanding the results given in the next section.

For an uniform and steady basic flow, $H=$ constant and $V=$ constant, one has $d \mathbf{S} / d X \equiv \mathbf{S}_{1}=0$ in (17) (18). In fact, the second equation in (17) becomes unnecessary, and the linear stability equation is just (13) with $d \mathbf{S} / d X=0$. The dispersion relation (22) reduces then to

$\operatorname{det}(\mathbf{A}+a \mathbf{B})=0$,

with $H_{X}=V_{X}=0$ in (14).

For an uniform and steady basic flow, the solution to (1)-(3) relates the constant values of $H$ and $V$ by

$H=\frac{f V^{2}}{8 \sin \theta}$

It is convenient to use the Froude number

$F r \equiv \frac{V}{c}=\frac{V}{\sqrt{H \cos \theta}}=\sqrt{\frac{8}{f} \tan \theta}$,

instead of the friction factor $f$, where $c=\sqrt{H \cos \theta}$ is the non-dimensional wave speed for small surface perturbations. Substituting (27)-(28) into (26), one obtains the following dispersion relation for the complex eigenvalue $a$ and the real frequency $\omega$ :

$$
\begin{gathered}
a^{2} V^{2}\left(1-\frac{1}{F r^{2}}\right)+a(3 \sin \theta-2 \omega V i) \\
-\omega^{2}-\frac{2 \sin \theta \omega}{V} i=0 .
\end{gathered}
$$

The number of parameters may be reduced to just one, the Froude number, if one makes the following changes of variables valid for $\sin \theta \neq 0$ :

$e \equiv \frac{a V^{2}}{\sin \theta}, \quad w \equiv \frac{\omega V}{\sin \theta}$,

so that (29) reduces to

$e^{2}(1-\varrho)+e(3-2 w i)-w^{2}-2 w i=0$,

where, for convenience, we use the inverse of the square of the Froude number,

$\varrho \equiv \frac{1}{F r^{2}}$.

The new growth rate $\sigma$ and wavenumber $\epsilon$ are defined as [see (12)]

$e \equiv \sigma+i \epsilon, \quad \sigma=\frac{\gamma V^{2}}{\sin \theta}, \quad \epsilon=\frac{\alpha V^{2}}{\sin \theta}$. 


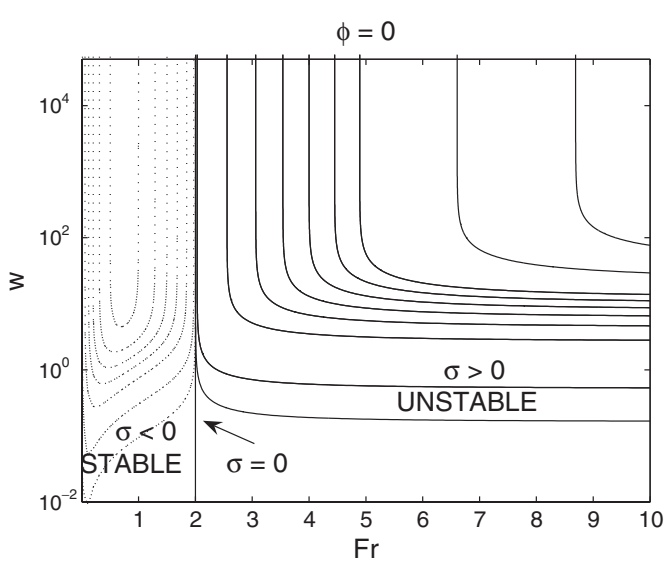

Figure 2. Contour lines for constant growth rate in the $(w, F r)$ plane for the parallel flow case. Continuous lines: $\sigma=0, .001, .01, .2, .4, .6, .8,1,1.2,2,3$. Dashed lines: $\sigma=-.001,-.01,-.05,-.1,-.15,-.2,-.25$.

The neutral curve for instability, corresponding $\sigma=0$, may be easily obtained by substituting $e=i \epsilon$ into (31). The imaginary and real parts of the resulting equation yield, respectively,

$\epsilon=\frac{2}{3} w \quad$ and $\quad \varrho=\frac{1}{4}$.

The flow is stable $(\sigma<0)$ for $\varrho>\varrho_{c}=1 / 4$, while it is (convectively) unstable $(\sigma>0)$ for any value of the frequency $w$ if $\varrho<1 / 4$. This obviously reproduces Jeffreys (1925) instability condition $\mathrm{Fr}>2$ : any perturbation is unstable above the critical Froude number 2. In addition, one obtains that the phase velocity of the neutrally stable waves,

$c_{p h} \equiv \frac{\omega}{\alpha}=\frac{w V}{\epsilon}=\frac{3}{2} V$,

is three times larger that the surface wave speed $c$ for the critical $F r=2, c=V / F r=V / 2$.

Figure 2 depicts the neutral curve in the plane $(F r, w)$, which is just the vertical line $F r=2$, together with some contour lines for constant growth rate $\sigma$. This figure qualitatively explains why Brock (1967) did not observed the formation of roll waves for Froude numbers below 3.5, approximately, in his experiments for an uniform flow in an inclined channel. For $\mathrm{Fr}$ close to the critical value $F r_{c}=2$ the growth rate is so small that a extremely long channel would be required for the developments of the waves (note that for $F r<3.5$, $\sigma<0.5$, approximately).

To finish this section it is worth to mention that for a horizontal channel $(\sin \theta=0)$, Eq. (29) does not yield unstable solutions (i.e., solutions with $\sigma>0$ together with $\epsilon>0$ ).

\subsection{Nonparallel flow: Kinematic wave approximation}

We now turn to the general nonparallel stability formulation (17). In order to obtain some quantitative results, but without recurring to any particular basic flow, we shall consider here the 'kinematic wave approximation' to the shallow-water flow equations (Lighthill and Whitham 1955; Whitham 1974), which have proved to be very useful for approximating the long time behavior of the flow down an open inclined channel (Hunt 1983).

In the kinematic wave approximation, one neglects the left hand side of Eq. (2) and, after using the friction law (3), obtains the same relation (27) between $H$ and $V$. But now, $H$ and $V$ are not constant, they must satisfy the continuity equation (1) and the initial and boundary condition of the particular problem under consideration. Substituting (27) into the left hand side of Eq. (2) and using (1), one obtains the following condition for the validity of this approximation:

$\left|\frac{\partial H}{\partial X}\right| \ll\left|\frac{\sin \theta}{\cos \theta-\frac{2}{f} \sin \theta}\right|$,

which in the case $f \ll 2 \tan \theta$ may be simplified to $|\partial H / \partial X| \ll f / 2$.

With this approximation, one can eliminate the dependence on $H$ and on $H_{X}$ of the dispersion relation (22) by using (27) and its derivative with respect to $X$ :

$H=\frac{f V^{2}}{8 \sin \theta}, \quad H_{X}=\frac{f V V_{X}}{4 \sin \theta}$.

In addition, the explicit dependence on the angle $\theta$ and on the local flow velocity $V$ can be eliminated from the dispersion relation by using the same change of variables (30) together with

$\phi \equiv \frac{V_{X} V}{\sin \theta}$.

Thus, taking the determinant of $\mathbf{F}$ (18) and using the above notation, the dispersion relation may be written as

$D(e, w ; \varrho, \phi) \equiv d_{0}+d_{1} \phi+d_{2} \phi^{2}+d_{3} \phi^{3}+d_{4} \phi^{4}=0$,

where

$$
\begin{aligned}
& d_{0} \equiv\left[e^{2}(\varrho-1)+w^{2}+2 i e w+(2 i w-3 e)\right]^{2}, \\
& d_{1} \equiv 2\left[3 i w^{3}+2 e^{3}(\varrho-1)^{2}+4 e w^{2}(\varrho-2)\right. \\
& \quad+7 i e^{2} w(\varrho-1)-13 e^{2}(\varrho-1)-12 w^{2} \\
& +2 i e w(5 \varrho-12)+(12 e-11 i w)]
\end{aligned}
$$




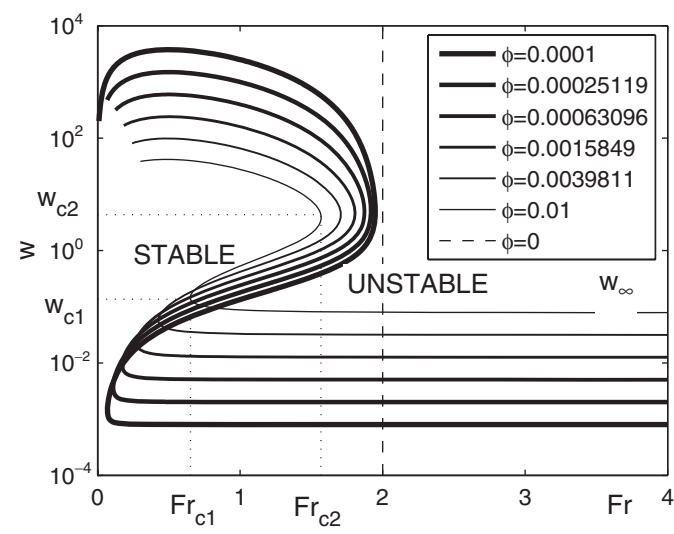

Figure 3. Neutral curves $(\sigma=0)$ for different values of $\phi>0$ (as indicated).

$$
\begin{aligned}
& d_{2} \equiv- 13 w^{2}+8 e^{2}(\varrho-1)^{2}+4 i e w(4 \varrho-5)+36 \\
& \quad-2[i w(27-4 \varrho)+e(22 \varrho-25)] \\
& d_{3} \equiv-4[3 i w+2 e(\varrho-1)+2(3 \varrho-5)] \\
& d_{4} \equiv 4
\end{aligned}
$$

and where use has been made of the Froude number (28) and (32). In the new variables, the validity condition (36) for the kinematic wave approximation reads

$|\phi| \ll\left|\frac{2}{4 \varrho-1}\right|$.

The neutral curves for instability, corresponding to vanishing real part of $e(\sigma=0)$ in (39), depend on $w$, $\varrho$ (or $\mathrm{Fr}$ ), and $\phi$. Figure 3 shows these neutral curves in the $(F r, w)$ plane for several values of $\phi>0$. As it is observed, there exists marked differences with the neutral curve for the parallel flow case $\phi=0$. Firstly, the flow is always stable for very small frequencies [for $w<w_{\infty}(\phi)$ ], independently of the Froude number. Secondly, the minimum, or critical, Froude number for instability, $F r_{c 1}(\phi)$, corresponding to the frequency $w_{c 1}(\phi)$, is always less that 2 when $\phi>0$. This critical Froude number tends to zero as $\phi$ decreases, though the frequency range around $w_{c 1}$ for which the flow is unstable is small, vanishing as $\phi \rightarrow 0$. As in the parallel case, the flow is unstable for almost any frequency when $F r>2$ (except for very small frequencies, as commented on above). In fact, there exists another critical value of the Froude number, $\operatorname{Fr}_{c 2}(\phi)<2$, corresponding to the frequency $w_{c 2}(\phi)$, above which the flow is always unstable provided that the frequency is not too small $\left(F r_{c 2} \rightarrow 2\right.$ and $w_{\infty} \rightarrow 0$ as $\left.\phi \rightarrow 0\right)$. For

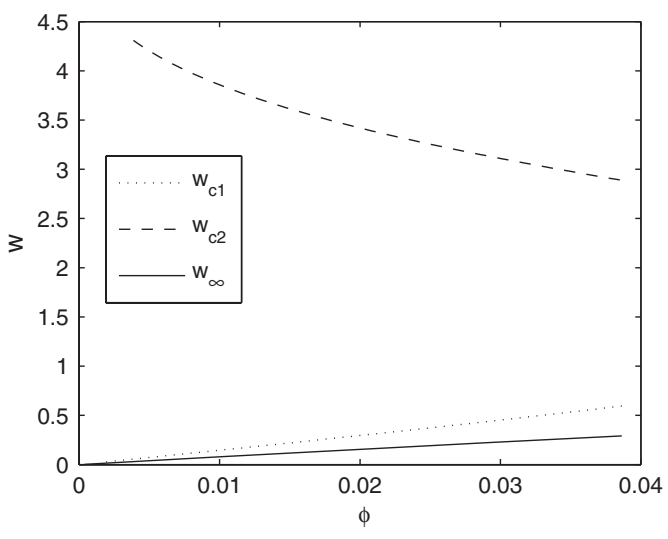

Figure 4. $w_{c 1}, w_{c 2}$, and $w_{\infty}$ as functions of $\phi$.

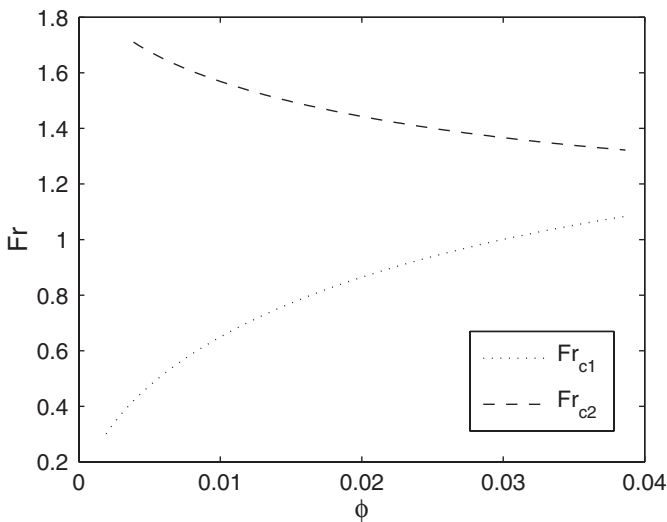

Figure 5. $F r_{c 1}$, and $F r_{c 2}$ as functions of $\phi$.

high frequencies the stability region shrinks to disappear for very high $w$, the larger the smaller $\phi$. But these very high frequencies, like the very small ones $w<w_{\infty}$, are too extreme to be physically meaningful. Thus, the two pairs of critical values $\left(F r_{c 1}, w_{c 1}\right)$ and $\left(F r_{c 2}, w_{c 2}\right)$ are the most significant physical results. Figures 4 and 5 show these critical values (together with $\left.w_{\infty}\right)$ as functions of $\phi(>0)$.

To have an idea of the most unstable frequencies, Fig. 6 shows contour lines of constant growth rate $\sigma$ for a particular value of $\phi$. It is observed that, though the critical $\mathrm{Fr}$ is much smaller than 2 for low frequencies, the growth rate for $\mathrm{Fr}<2$ is always quite small (except for very high, unrealistic frequencies). This explains why we have to use a very long channel and leave the flow evolve during a quite long time in our numerical simulations of the next section to observe the formation of roll waves for $F r<2$.

In all the numerical computations we have made where roll waves are formed in a nonuniform flow (some of them reported in the next section for the 


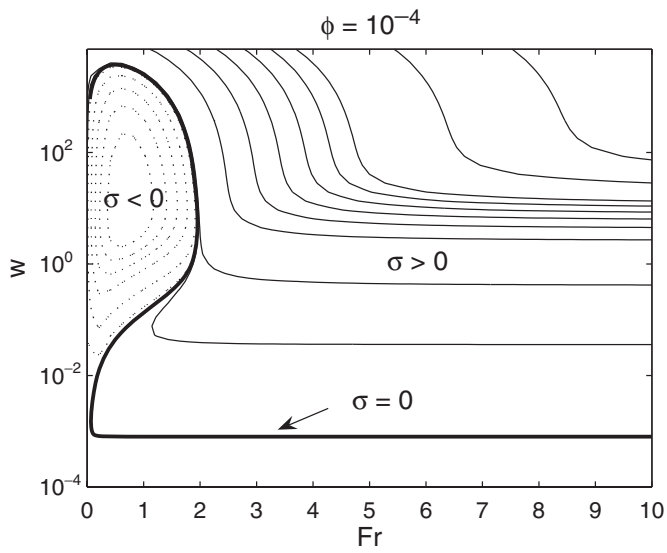

Figure 6. Contour lines of constant growth rate in the $(w, F r)$ plane for $\phi=10^{-4}$. Continuous lines: $\sigma=0, .001, .01, .2, .4$, $.6, .8,1,1.2,2,3$. Dashed lines: $\sigma=-.001,-.01,-.05,-.1$, $-.15,-.2$.

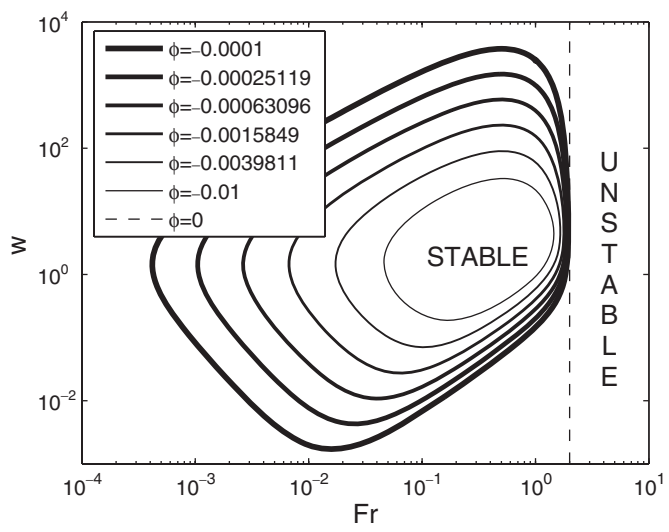

Figure 7. Neutral curves $(\sigma=0)$ for different values of $\phi<0$ (as indicated).

dam-break problem on an inclined channel), the waves develop in regions of the flow where the kinematic wave approximation applies and where $\phi>0$, i.e. in regions where the velocity (and the height) increases downstream. Nonetheless, we include here the stability results for $\phi<0$ for completeness. Figure 7 shows the neutral curves in the $(F r, w)$ plane for several values of $\phi<0$, while Fig. 8 depicts contour lines of constant growth rate $\sigma$ for a particular value of $\phi<0$.

As in the cases with $\phi>0$, the flow may be unstable for $\mathrm{Fr}<2$ and the stability properties are very sensitive to the non-uniformities of the flow: with very small values of $|\phi|$, the region of instability changes dramatically with respect to the parallel flow case. However, the growth rates for $\mathrm{Fr}<2$ are very small, except for

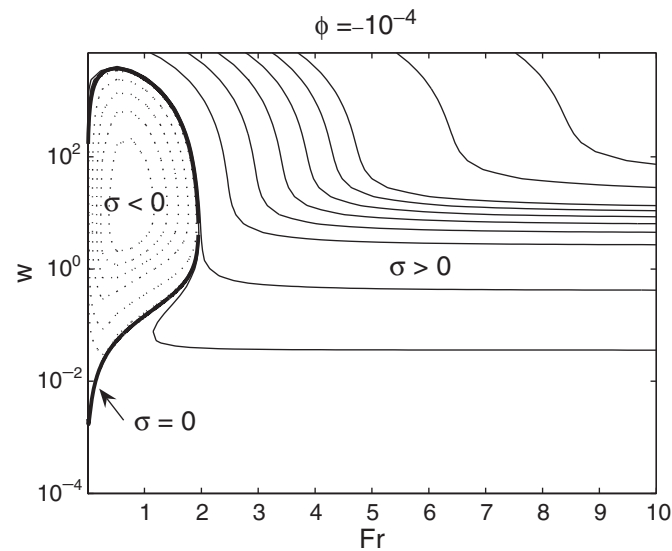

Figure 8. As in Fig. 6, but for $\phi=-10^{-4}$.

very high unrealistic frequencies (Fig. 8), as it also occurs for $\phi>0$. There are, nonetheless, some important differences between $\phi>0$ and $\phi<0$. First, for $\phi<0$ there no exists the frequency $w_{\infty}$, and the flow is always unstable as $w \rightarrow 0$. In fact, the stable region in the $(F r, w)$ plane is closed, so that the flow is unstable for any frequency when $\mathrm{Fr}$ is very small (Fig. 7). However, for these very small values of the Froude number the kinematic wave approximation is no longer valid because condition (45) implies

$F r \gg \sqrt{\frac{4|\phi|}{2+|\phi|}} \simeq \sqrt{2|\phi|}$.

This applies for both $\phi<0$ and $\phi>0$. This restriction, together with the very small growth rates for $\mathrm{Fr}<2$ in both cases, explain why it is difficult to observe the development of roll waves when $\mathrm{Fr}<2$, in spite of the fact that the critical Froude number for instability can be much smaller than 2 for both positive and negative values of $\phi$.

\section{COMPARISON WITH NUMERICAL RESULTS FOR THE DAM-BREAK PROBLEM}

As an example on the formation of roll waves in a nonuniform flow, we have considered the dam-break problem in an inclined channel (Fernandez-Feria 2006; Bohorquez and Fernandez-Feria 2006). Actually, our interest in the present stability problem originated from the observation of roll waves in some numerical simulations for that non-uniform flow problem. Basically, a finite mass of water like that sketched in the inset of Fig. 9 is released at $t=0$ on an inclined plane of angle $\theta$. The equations (1)-(2)are solved numerically by using a high-resolution TVD finite-volume 


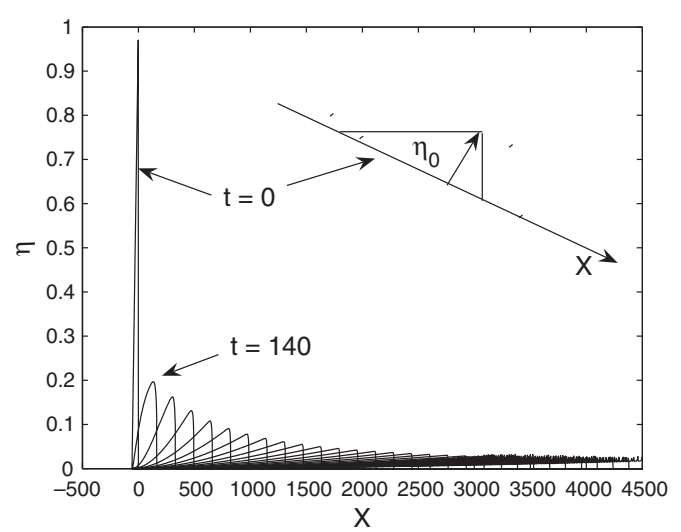

Figure 9. Water depth $\eta(X)$ for different instants of time in the dam break problem for $\theta=1^{\circ}$ and $F r=2.5$. The different curves are for $t=0,140,288,454,638,841,1061$, $1298,1550,1817,2097,2392,2699,3018,3349,3691,4045$, $4409,4784,5167,5542,5901,6267,6637,7012,7394,7779$, $8172,8568,8966$. Also included is a sketch of the initial configuration of the dam-break problem.

method like that described in Burgete and GarciaNavarro (2001) (see the above mentioned references for the details of the formulation of the problem and the numerical method used). We have started a systematic search for the instabilities and the formation of roll waves in that flow, and we present here some preliminary results.

We have not found the spontaneous formation (i.e., with the only forcing of the round-off numerical noise) of roll waves in our numerical simulations for Froude numbers less than 2 . This is explained by the small growth rate of the unstable perturbations when $\mathrm{Fr}$ is less than, approximately, 2 (see Figs. 6 and 8), so that a very long channel is needed for the roll waves to develop. In fact, we have observed perturbations growing downstream for $\mathrm{Fr}<2$ when disturbances with much higher amplitude than the numerical noise are introduced upstream. But these waves never reach the stage of a roll wave for $\mathrm{Fr}<2.5$ with the channel lengths used so far. In any case, to our knowledge, no roll waves have been documented before from experimental observations for Froude numbers less than approximately 3.5 , whence the interest of the numerical results given below.

Figure 9 shows the evolution of the water depth $\eta$ for several instants of time after the break of the dam in a channel with $\theta=1^{\circ}$ and for $F r=2.5$ (a sketch of the initial configuration is also shown in the figure). For $t \simeq 4400$, the instability in the free surface begins to be appreciated in the numerical simulations, originated just from numerical noise, as it is clearly evident in Fig. 10 for $t=4784$. For these instants of time, the kinematic wave approximation is already

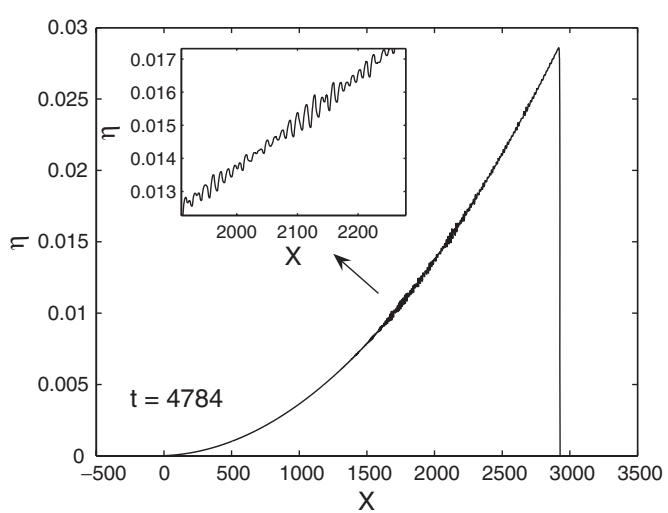

Figure 10. $\eta(X)$ for $t=4784$ (from Fig. 9).

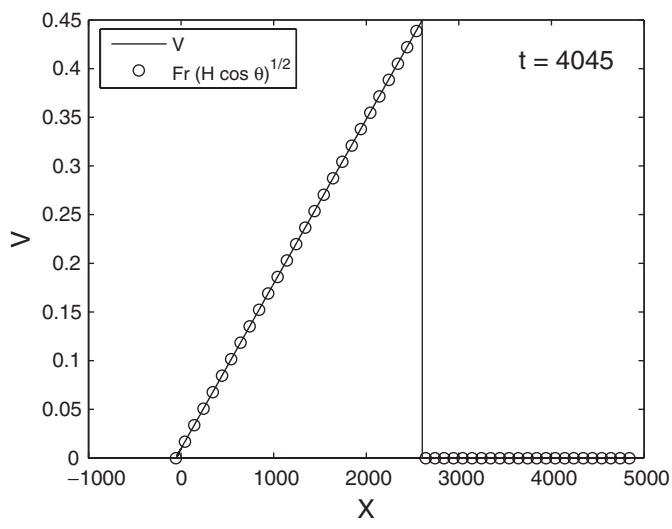

Figure 11. Comparison between the numerical results for the velocity (line) and the kinematic wave approximation (circles), for the flow depicted in Fig. 9 at $t=4045$.

valid in all the flow domain (in fact, it is very accurate; see Fig. 11), so that one may use the stability results obtained in the last section. Eventually, the amplitude of the instability waves grows to reach the stage where the free surface has the aspect of the classical roll waves (see Fig. 12).

The frequency of the initial waves developed after the instability of the flow can be obtained by plotting the velocity perturbation at a given location as a function of time. Figure 13 is such a plot for $X=2342$, starting from $t=4400$, the instant at which one begins to observe numerically the formation of the instability waves at the surface. The velocity perturbation, $U^{\prime}=U-V$, has a frequency $\omega \simeq 0.1924$, which remains almost constant for a long period of time. Taking into account that during the period of time considered in Fig. $13 \mathrm{~V}$ and its gradient vary, in the location considered, in the ranges $0.28<V<0.37$ and $1.9 \times 10^{-3}<\phi<3.3 \times 10^{-3}$, respectively, that 


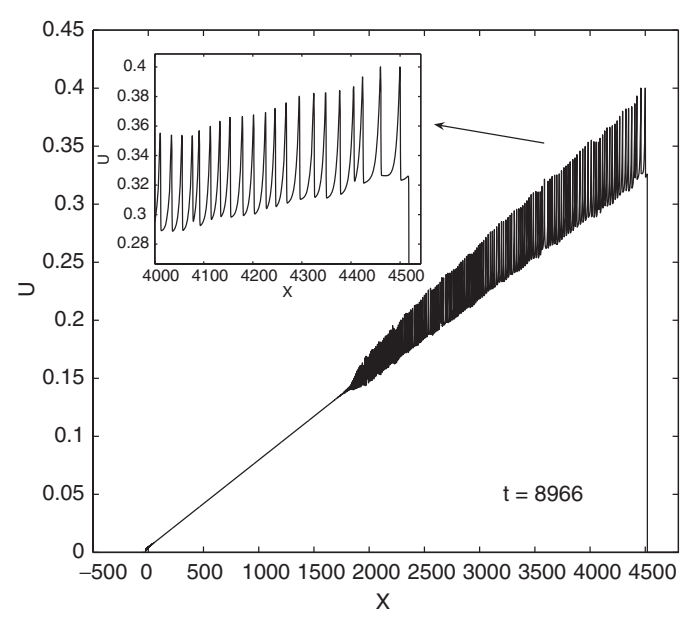

Figure 12. $U(X)$ at $t=8966$ for the flow depicted in Fig. 9.

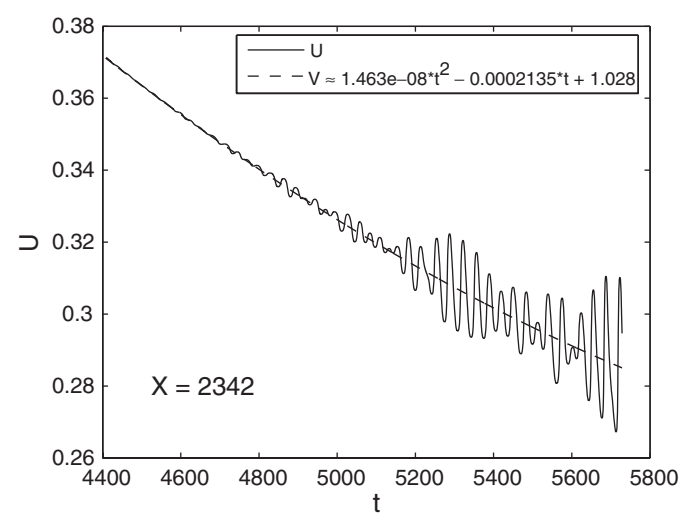

Figure 13. $U(t)$ at $X=2342$ for the flow depicted in Fig. 9 . Also shown is an extrapolation of the mean velocity without perturbation, $V(t)$ (dashed line).

frequency corresponds to $3.1<w<4.1$, approximately. For these values of $w, \phi$ and $F r$, the stability analysis predicts that the eigenvalue $e=\sigma+\epsilon i$ ranges between $0.1136+2.163 i$ and $0.1591+2.8922 i$ $(0.0203+0.3687 i$ and $0.0253+0.4814 i$ in terms of $a=\gamma+\alpha i)$. This corroborates, first, that the growth rate $\sigma$ cannot be too small in order to observe the instabilities in the numerical simulations ( $\sigma$ is larger that 0.1 , which in Fig. 6 only occurs if $\mathrm{Fr}$ is sufficiently larger than 2, if the frequency $w$ is not too large). On the other hand, the wavelength $\lambda=2 \pi / \alpha$ given by the stability analysis ranges between 13 and 17, approximately. This agrees quite well with the wavelength in the numerical simulation, which from the inset in Fig. 10 can be estimated as the ratio between the space domain length to the number of crests to be $370 / 27 \approx 14$.

\section{CONCLUSIONS}

We have analysed the spatial stability of the shallowwater flow in an inclined surface of arbitrary slope. We have developed a local analysis that takes into account the effect of the local gradients of both the velocity and the water depth. In particular, we have considered the kinematic wave approximation, that is shown to be a very good approximation to any non-uniform and non-steady frictional flow for sufficiently long times. The stability results depend only on two dimensionless parameters: the Froude $(\mathrm{Fr})$ number and a parameter $\phi$ characterizing the local gradient of the water depth. Since the stability analysis is a spatial one, we have characterized the frequency ranges in the parameter space $(F r, \phi)$ for which instabilities develop. The main result is that the stability properties of the flow change qualitatively (substantially) when the non-parallelism of the shallow-water flow is taken into account. In particular, instabilities can be present for Froude numbers smaller than 2 in some frequency ranges that depends on $\phi$ (for a parallel, constant-depth flow, the instability condition is $F r>2$ ). However, the growth rate for the unstable modes is so small when $\mathrm{Fr}<2$ that, without artificially disturbing the initial flow, we have not observed the formation of roll waves in our numerical simulations for the dam-break problem in a inclined surface when $F r<2$. We have been able to simulate numerically the formation of roll waves in the dambreak problem in an inclined surface for $F r=2.5$. The instability waves, and the subsequent roll waves, develop from just the numerical round-off noise at this Froude number, and for larger values of $F r$. This is also a relevant finding because, to our knowledge, there are no previous observations of roll waves in physical experiments for $\mathrm{Fr}$ less than approximately 3.5, due to the above mentioned fact that the growth rate of the perturbations is very small when $\mathrm{Fr}$ is close to its critical value for instability. Finally, we find that the reported numerical results compare very well with the predictions of the stability analysis.

\section{REFERENCES}

Berlamont, J. E. and N. Vanderstappen (1981). Unstable turbulent flow in open channels. J. Hydraul. Div. 107, HY4, 427-449.

Bertolotti, F. P., T. Herbert, and P. R. Spalart (1992). Linear and nonlinear stability of the blasius boundary layer. J. Fluid Mech. 242, 441-474.

Bohorquez, P. and R. Fernandez-Feria (2006). Transport of suspended sediment under the dam-break flow on an inclined bed of arbitrary slope. J. Hydr. Res., submitted. 
Bouchut, F., A. Mangeney-Castelnau, B. Perthame, and V. J. P. (2003). A new model of Saint Venant and Savage-Hutter type for gravity driven shallow water flows. C. R. Acad. Sci. Paris, Ser. I 336, 531-536.

Brock, R. R. (1967). Development of roll waves in open channels. Technical report, W. M. Keck Lab. of Hydraul. and Water Resources, California Inst. of Tech., Report KH-R-16.

Burguete, J. and P. Garcia-Navarro (2001). Efficient construction of high-resolution TVD conservative schemes for equations with source terms: application to shallow water flows. Int. J. Numer. Meth. Fluids 37, 209-248.

Chanson, H. (2004). The hydraulics of open channel flow: an introduction. Elsevier, Amsterdam.

Cornish, V. (1934). Ocean waves and kindred geophysical phenomena. Cambridge University Press.

Di Cristo, C. and A. Vacca (2005). On the convective nature of roll waves instability. J. Appl. Math. 2005:3, 259-271.

Dressler, R. F. (1949). Mathematical solution of the problem of roll waves in inclined open channels. Commun. Pure Appl. Maths. 2, 149-194.

Fernandez-Feria, R. (2000). Axisymmetric instabilities of Bodewadt flow. Phys. Fluids 12, 1730-1739.
Fernandez-Feria, R. (2006). Dam-break flow for arbitrary slopes of the bottom. J. Engng. Math., in press (published online).

Hunt, B. (1983). Asymptotic solution for dam break on a sloping channel. J. Hydraulic Eng. 109, 1698-1706.

Jeffreys, H. (1925). The flow of water in an inclined channel of rectangular section. Phil. Mag. 49, 793-807.

Keller, J. B. (2003). Shallow-water theory for arbitrary slopes of the bottom. J. Fluid Mech. 489, 345-348.

Kenyon, K. E. (1998). Roll wave theory. Phys. Essays 11, 531-540.

Lighthill, M. J. and G. B. Whitham (1955). On kinematic waves: I. Flood movement in long rivers; II. Theory of traffic flow on long crowed roads. Proc. Roy. Soc. A, 229, 281-345.

Savage, S. B. and K. Hutter (1991). The dynamics of avalanches of granular materials from initiation to run-out. Acta Mech. 86, 201-223.

Stoker, J. J. (1957). Water waves. Interscience.

Whitham, G. B. (1974). Linear and nonlinear waves. Wiley, New York. 